\title{
TELESSAÚDE NO CONTEXTO DA PANDEMIA DA COVID-19: REVISÃO DE ESCOPO
}

\section{TELEHEALTH IN THE CONTEXT OF THE COVID-19 PANDEMIC: A SCOPING REVIEW}

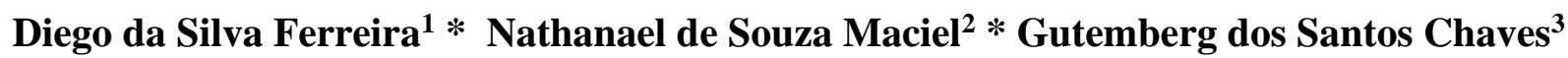 \\ Davide Carlos Joaquim ${ }^{4} *$ Francisco Jardsom Moura Luzia $^{5} *$ Edmara Chaves Costa $^{6}$
}

\section{RESUMO}

Objetivo: Analisar as publicações científicas disponíveis na literatura que descrevem a telessaúde no contexto da COVID-19. Métodos: Revisão de escopo da literatura seguindo seis etapas. A pergunta norteadora foi: "Quais as publicações científicas disponíveis na literatura que descrevem a telessaúde no contexto da COVID-19?". Utilizou-se os descritores "COVID-19", "Coronavirus" e "Telemedicine". A pesquisa ocorreu nas seguintes bases: MEDLINE, CINAHL, SCOPUS, COCHRANE, WOS, LILACS, SCIELO e PUBMED. Resultados: Foram identificados 474 artigos nas buscas nas bases de dados, após a análise, resultou-se uma amostra de 31 artigos. Foram organizadas as seguintes categorias: recursos tecnológicos; potencial e benefícios; desafios e obstáculos; e estratégias de implementação. Conclusão: A telessaúde pode ampliar o acesso e levar assistência remota sem expor os profissionais da saúde, reduzindo o risco de contágio à COVID-19. Palavras-chave: Telemedicina; Infecções por Coronavirus; Pandemia; Serviços de Saúde; Tecnologia da Informação.

\section{ABSTRACT}

Objective: To analyze the scientific publications available in the literature that describe the telehealth in the context of COVID-19. Methods: Literature scope review following six steps. The guided question was: "What scientific publications are available in the literature that describe the telehealth in the context of COVID-19?". The descriptors "COVID-19", "Coronavirus" and "Telemedicine" were used. The research took place in the following databases: MEDLINE, CINAHL, SCOPUS, COCHRANE, WOS, LILACS, SCIELO and PUBMED. Results: A sample of 31 articles was identified in the searches in the databases, and a sample of 31 articles was resulted. The following categories were organized: technological resources; potential and benefits; challenges and obstacles; implementation strategies. Conclusion: Telemedicine can expand access and provide remote care without exposing health professionals, reducing the risk of contagion to COVID-19.

Keyword: Telemedicine; Coronavirus Infections; Pandemics; Health Services; Information Technology.

\footnotetext{
${ }^{1}$ Enfermeiro. Doutorando em Saúde Coletiva. Mestre em Enfermagem. Universidade Estadual do Ceará. Fortaleza, CE, Brasil. Orcid id: https://orcid.org/0000-0002-6314-5405 E-mail: diegoferreira@aluno.unilab.edu.br

Telefone: (85)981903676

${ }^{2}$ Discente do curso de Bacharelado em Enfermagem. Universidade da Integração Internacional da Lusofonia Afro-Brasileira. Redenção, CE, Brasil. Orcid id: https://orcid.org/0000-0002-5088-011X E-mail: nathanael.souza.inf@ gmail.com

3 Enfermeiro. Especialista em Saúde da Família pela Universidade da Integração Internacional da Lusofonia Afro-Brasileira. Redenção, CE, Brasil. Orcid id: https://orcid.org/0000-0002-6994-243X E-mail: gutembergchave@gmail.com

${ }^{4}$ Enfermeiro. Doutorando em Ciências Morfofuncionais. Mestre em Enfermagem. Universidade Federal do Ceará. Fortaleza, CE, Brasil. Orcid: https://orcid.org/0000-0003-0245-3110 E-mail: davidejoaquim@ hotmail.com

5 Discente do curso de Bacharelado em Enfermagem. Universidade da Integração Internacional da Lusofonia Afro-Brasileira. Redenção, CE, Brasil. Orcid id: https://orcid.org/0000-0002-8386-6103 E-mail: jaarmoura@ gmail.com

${ }^{6}$ Médica veterinária. Docente do curso de Bacharelado em Enfermagem da Universidade da Integração Internacional da Lusofonia Afro-Brasileira. Redenção, CE, Brasil. Orcid: https://orcid.org/0000-0003-0007-6681 E-mail: edmaracosta@unilab.edu.br
} 


\section{INTRODUÇÃO}

A doença COVID-19 é uma infecção respiratória que pode apresentar um quadro clínico assintomático a até quadros respiratórios graves, causada por um vírus do grupo dos coronavírus (SARS-CoV-2), transmitida pelo contato próximo e desprotegido com secreções ou excreções de um paciente infectado, principalmente por meio de gotículas salivares ${ }^{(1)}$. A transmissão também pode ocorrer pelo contato com objetos ou superfícies contaminadas ${ }^{(1-2)}$. Além disso, as evidências sugerem que a via fecal-oral pode ser uma forma da transmissão do vírus $^{(2)}$.

Desde a sua origem na cidade de Wuhan, na China, a COVID-19 tornou-se um problema de saúde pública de preocupação mundial que está ameaçando seriamente a saúde das pessoas em todo o mundo. De fato, a sua repercussão transpassa a higidez fisiológica do indivíduo, estendendo-se aos aspectos psicossociais, econômicos e políticos ${ }^{(2)}$. De acordo com os dados da Organização Mundial de Saúde, até 2 de fevereiro de 2021, existiam 102139771 casos confirmados e 2211762 mortes no mundo ${ }^{(3)}$. No Brasil, a doença é responsável por 9118513 casos confirmados em todo território nacional e 222666 mortes $^{(3)}$.

Os impactos e a gravidade da COVID-19 permanecem indefinidos. Sabe-se que os pacientes infectados podem apresentar sintomas leves (febre, tosse, dispneia, mialgias e fadiga), graves e críticos (pneumonia viral e podem evoluir para situações de síndrome de dificuldade respiratória aguda, insuficiência cardíaca aguda, lesão renal aguda, sepse ou choque $)^{(2,4)}$. As evidências disponíveis indicam que idosos, pessoas com doença cardiovascular, diabetes mellitus, hipertensão arterial sistêmica, doença pulmonar crônica, doença renal crônica e neoplasia apresentam o risco de evoluírem para infecção grave e pacientes pré-sintomáticos podem apresentar um potencial risco para propagação da infecção ${ }^{(4)}$.

A pandemia de COVID-19 representa um enorme desafio para os sistemas de saúde globalmente, impondo demandas excessivas que ocasionam superlotação das unidades de assistência à saúde, prejudicando de forma severa a capacidade de resposta a prestação de cuidados, bem como recursos humanos treinados ${ }^{(5)}$, além de obstaculizar o atendimento de pacientes com outras patologias, devido alto risco da transmissão do vírus ${ }^{(6)}$. Ademais, a pandemia evidenciou as disparidade econômicas, onde os mais pobres sofreram impactos que ainda não conseguem ser mensurados ${ }^{(7)}$. Neste contexto, práticas inovadoras de atendimento de forma remota têm emergido, tornando pertinente o uso das tecnologias de comunicação a fim de minimizar esses impactos.

Compreende-se telemedicina como um conjunto de recursos tecnológicos de comunicação eletrônica (smartphones, tablets de computador, aplicativos móveis e videoconferência) utilizadas para fornecer serviços e educação em saúde de modo à distância e se constitui principal forma de enfrentar os desafios relacionados a escassez de profissionais de saúde e de infraestruturas ${ }^{(8)}$, além de fornecer suporte clínico, transpor barreiras geográficas, integrar a utilização de variadas tecnologias e melhorar os resultados de saúde ${ }^{(9)}$. A telemedicina e a telessaúde representam oportunidades para melhorar o acesso à assistência de saúde durante a pandemia da COVID-19, uma vez que permite 
estender os serviços de saúde para regiões que não possuem assistência, bem como evitar o contato físico entre o profissional e pacientes, assim, reduzir o risco de contaminação ${ }^{(1,8)}$.

Diante disso, pretende-se com este estudo ampliar a visibilidade do uso da telessaúde durante as crises de saúde pública, contribuindo para nortear e fornecer subsídios para os profissionais da saúde sobre a possibilidade de estratégias a serem adotas na prática assistencial, assim como apresentar uma reflexão que possa contribuir na melhoria do acesso aos serviços de saúde, ampliar o conhecimento a respeito do que foi produzido cientificamente e relevância para atualidade sobre a temática. O objetivo deste estudo é analisar as publicações científicas disponíveis na literatura que descrevem a telessaúde no contexto da COVID-19.

\section{MÉTODOS}

Trata-se de uma revisão de escopo. O objetivo dessa revisão é mapear, através de um método consistente, o estado da arte em um determinado assunto, com o objetivo de mostrar uma visão descritiva dos estudos revisados ${ }^{(10)}$.

Para sistematização dos dados foi utilizado o Preferred Reporting Items for Systematic reviews and Meta-Analyses extension for Scoping Reviews (PRISMA-ScR) Checklist, um guia para escrever uma revisão de escopo. $\mathrm{O}$ PRISMA-ScR é formado por 22 itens categorizados em: Título, Resumo, Introdução, Método, Resultados, Discussão e Financiamento $^{(11)}$.

Nessa perspectiva, esta revisão de escopo usou o arcabouço metodológico proposto por Joanna Briggs Institute. A estrutura desta revisão foi dividida em seis etapas: 1) estabelecimento da questão e objetivo de pesquisa; 2) assimilação de estudos relevantes, que atendessem o alvo da revisão; 3) escolha de estudo, conforme os critérios estabelecidos; 4) mapeamento de dados; 5) organização dos resultados, por meio da a análise qualitativa em relação ao objetivo e pergunta; 6) apresentação dos resultados ${ }^{(10)}$.

Nesse sentido, construiu-se a seguinte questão norteadora: "Quais as publicações científicas disponíveis na literatura que descrevem a telessaúde no contexto da COVID-19?".

Utilizou-se os descritores controlados "COVID-19", "Coronavirus" e "Telemedicine", especificados no Medical Subject Headings (MeSH). Usou-se o operador booleano AND nas seguintes combinações: "Coronavirus" AND "Telemedicine"; "COVID-19" AND "Telemedicine".

A pesquisa ocorreu em maio de 2020 nas seguintes bases: Medical Literature Analysis and Retrieval System Online (MEDLINE), Cummulative Index to Nursing and Allied Health Literature (CINAHL), Scopus, Cochrane, Web of Science (WOS), Literatura Latino-Americana e do Caribe em Ciências da Saúde (Lilacs), Scientific Electronic Library Online (SciELO) e PUBMED.

Foram incluídos artigos publicados em português, inglês e espanhol. Foram excluídos os artigos de revisão, estudos reflexivos, resenhas, dissertações, monografas, teses, resumos em anais de eventos, artigos duplicados ou que não abordassem a temática relevante ao objetivo da revisão. Mantiveram-se os editoriais e relatos de experiência em razão da escassez de produção sobre a temática. A pré-seleção dos artigos ocorreu por meio da leitura do título, resumo e descritores e a seleção dos artigos pela leitura na íntegra. 
Foram identificados 474 artigos nas buscas empregadas nas bases de dados selecionadas para a condução da revisão, sendo excluídas 247 publicações duplicadas e, após a leitura de títulos, resumos e descritores, 122 artigos foram analisados na íntegra. Desses, 91 artigos completos foram excluídos por motivos diversos, a serem detalhados na sequência. $\mathrm{Na}$ Figura 1 é apresentado o processo de seleção dos estudos para compor a revisão.

Figura 1 - Fluxograma de identificação do processo de seleção dos estudos para compor a revisão de escopo. Redenção, CE, Brasil, 2020.

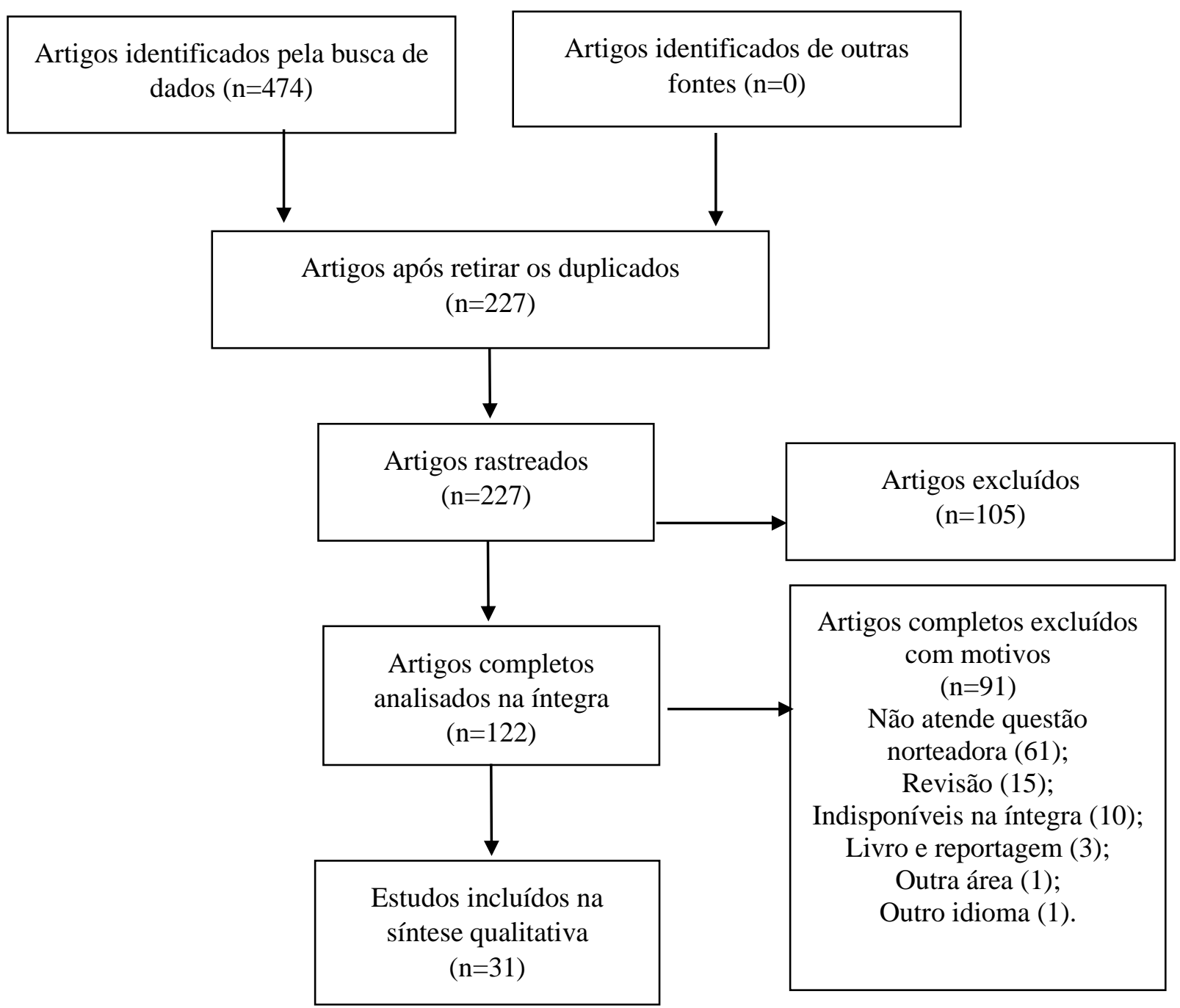

Para extração dos dados, utilizou-se uma planilha que permite a identificação da pesquisa, autores, local, data, intervenção e especialidade. Os dados foram armazenados em um banco de dados no software Microsoft Office Excel® versão 2016 e apresentados em quadros sinópticos e os resultados foram agrupados em quatro categorias: recursos tecnológicos; potencial e benefícios; desafios e obstáculos; estratégias de implementação.

\section{RESULTADOS}

A amostra deste estudo foi composta por 31 artigos. Quanto ao desenho do estudo, 12 $(38,71 \%)$ eram editoriais ou cartas, $8(25,81 \%)$ artigos originais, $6(19,35 \%)$ relatos de 
experiências, $3(9,68 \%)$ artigos de opinião e 2 $(6,45 \%)$ relatos de caso. Quanto à origem dos artigos 15 (48,39\%) eram dos EUA. Com relação à base de dados, 17 (54,84\%) eram da MEDLINE. No que se refere aos participantes do estudo, apenas $10(32,26 \%)$ incluem enfermeiros, sejam diretamente nomeados ou indiretamente como profissionais de saúde ou equipe multiprofissional. Todos os artigos foram publicados em 2020. Tabela 1 apresenta dados sobre os artigos identificados.

Tabela 1 - Caracterização dos artigos selecionados. Redenção, CE, Brasil, 2020

\begin{tabular}{|c|c|c|c|c|c|}
\hline & Periódico & Autores & Título & Base & Local \\
\hline & $\begin{array}{l}\text { Otolaryngolog } \\
\mathrm{y}-\text { Head and } \\
\text { Neck Surgery }\end{array}$ & $\begin{array}{l}\text { Triantafillou, } \\
\text { Rajasekaran }^{(6)}\end{array}$ & $\begin{array}{l}\text { A commentary on the challenges of } \\
\text { telemedicine for head and neck } \\
\text { oncologic patients during COVID- } \\
19 \text {. }\end{array}$ & MEDLINE $^{*}$ & $\mathrm{EUA}^{\dagger}$ \\
\hline 2 & $\begin{array}{l}\text { JMIR Public } \\
\text { Health } \\
\text { Surveillance }\end{array}$ & $\begin{array}{l}\text { Zamberg, et } \\
\text { al. }^{(13)}\end{array}$ & $\begin{array}{l}\text { A mobile health platform to } \\
\text { disseminate validated institutional } \\
\text { measurements during the COVID- } \\
19 \text { outbreak: utilization-focused } \\
\text { evaluation study. }\end{array}$ & MEDLINE & Suíça \\
\hline 3 & $\begin{array}{l}\text { European } \\
\text { Journal of } \\
\text { Cancer }\end{array}$ & Scotté, et al. ${ }^{(14)}$ & $\begin{array}{l}\text { A patient reported outcome } \\
\text { platform, a useful tool to improve } \\
\text { monitoring and effective } \\
\text { management of COVID-19- } \\
\text { positive patients with cancer }\end{array}$ & SCOPUS & França \\
\hline 4 & $\begin{array}{l}\text { Alcoholism \& } \\
\text { Drug Abuse } \\
\text { Weekly }\end{array}$ & Knopf $^{(15)}$ & $\begin{array}{l}\text { Addiction telemedicine comes into } \\
\text { its own with COVID- } 19 \text {. }\end{array}$ & CINAHL $^{\ddagger}$ & EUA \\
\hline 5 & $\begin{array}{l}\text { Journal of } \\
\text { Clinical } \\
\text { Virology }\end{array}$ & $\begin{array}{l}\text { Moazzami, et } \\
\text { al. }{ }^{(8)}\end{array}$ & $\begin{array}{l}\text { COVID-19 and telemedicine: } \\
\text { Immediate action required for } \\
\text { maintaining healthcare providers } \\
\text { well-being. }\end{array}$ & MEDLINE & Irã \\
\hline 6 & $\begin{array}{l}\text { Jounal of The } \\
\text { American } \\
\text { Medical } \\
\text { Informatics } \\
\text { Association }\end{array}$ & Mann, et al. ${ }^{(16)}$ & $\begin{array}{l}\text { COVID-19 transforms health care } \\
\text { through telemedicine: evidence } \\
\text { from the field. }\end{array}$ & MEDLINE & EUA \\
\hline 7 & $\begin{array}{l}\text { The Journal of } \\
\text { The American } \\
\text { Academy of } \\
\text { Orthopaedic } \\
\text { Surgeons }\end{array}$ & Loeb, et al. ${ }^{(17)}$ & $\begin{array}{l}\text { Departmental experience and } \\
\text { lessons learned with accelerated } \\
\text { introduction of telemedicine during } \\
\text { the COVID-19 Crisis. }\end{array}$ & MEDLINE & EUA \\
\hline 8 & AIDS and & Rogers, & $\begin{array}{l}\text { Development of telemedicine } \\
\text { infrastructure at a clinic to support }\end{array}$ & MEDLINE & EUA \\
\hline
\end{tabular}




\begin{tabular}{|c|c|c|c|c|c|}
\hline & Behavior & al. $^{(18)}$ & $\begin{array}{l}\mathrm{HIV}^{\S} \text { prevention and care in } \\
\text { response to } \\
\text { Providence. }\end{array}$ & & \\
\hline 9 & $\begin{array}{l}\text { American } \\
\text { Journal of } \\
\text { Otolaryngolog } \\
y\end{array}$ & $\begin{array}{l}\text { Pollock, } \\
\text { Setzen, } \\
\text { Svider }^{(19)}\end{array}$ & $\begin{array}{l}\text { Embracing telemedicine into your } \\
\text { otolaryngology practice amid the } \\
\text { COVID-19 crisis: An invited } \\
\text { commentary }\end{array}$ & MEDLINE & EUA \\
\hline 10 & $\begin{array}{l}\text { Telemedicine } \\
\text { and e-Health }\end{array}$ & $\begin{array}{l}\text { Huang, et } \\
\text { al. }{ }^{(20)}\end{array}$ & $\begin{array}{l}\text { Implications } \quad \text { for } r \\
\text { management: Two cases with } \\
\text { COVID-19 }\end{array}$ & WOS" & China \\
\hline 11 & $\begin{array}{l}\text { Journal of } \\
\text { Medical } \\
\text { Internet } \\
\text { Research }\end{array}$ & Gong, et al..$^{(21)}$ & $\begin{array}{l}\text { Internet hospitals help prevent and } \\
\text { control the epidemic of COVID-19 } \\
\text { in China: Multicenter user profiling } \\
\text { study }\end{array}$ & WOS & China \\
\hline 12 & Urology & $\begin{array}{l}\text { Borchert, et } \\
\text { al. }{ }^{(22)}\end{array}$ & $\begin{array}{l}\text { Managing urology consultations } \\
\text { during COVID-19 pandemic: } \\
\text { application of a structured care } \\
\text { pathway. }\end{array}$ & MEDLINE & EUA \\
\hline 13 & $\begin{array}{l}\text { Journal } \\
\text { francais } \\
\text { d'ophtalmolog } \\
\text { ie }\end{array}$ & $\begin{array}{l}\text { Daruich, } \\
\text { Martin, } \\
\text { Bremond- } \\
\text { Gignac }^{(23)}\end{array}$ & $\begin{array}{l}\text { Ocular manifestation as first sign of } \\
\text { Coronavirus Disease } 2019 \\
\text { (COVID-19): Interest of } \\
\text { telemedicine during the pandemic } \\
\text { context. }\end{array}$ & MEDLINE & Argentina \\
\hline 14 & $\begin{array}{l}\text { Rhode Island } \\
\text { Medical } \\
\text { Journal }\end{array}$ & Korr $^{(24)}$ & $\begin{array}{l}\text { On the front lines of primary care } \\
\text { during the coronavirus pandemic } \\
\text { shifting from office visits to } \\
\text { telephone triage, telemedicine. }\end{array}$ & MEDLINE & EUA \\
\hline 15 & $\begin{array}{l}\text { Telemedicine } \\
\text { and e-Health }\end{array}$ & $\begin{array}{l}\text { Al Kasab, } \\
\text { Almallouhi, } \\
\text { Holmstedt }^{(25)}\end{array}$ & $\begin{array}{l}\text { Optimizing the use of } \\
\text { teleneurology during the COVID- } \\
19 \text { pandemic. }\end{array}$ & MEDLINE & EUA \\
\hline 16 & $\begin{array}{l}\text { Journal of } \\
\text { Pain and } \\
\text { Symptom } \\
\text { Manegement }\end{array}$ & $\begin{array}{l}\text { Humphreys, et } \\
\text { al. }{ }^{(26)}\end{array}$ & $\begin{array}{lr}\text { Rapid implementation of inpatient } \\
\text { telepalliative } & \text { medicine } \\
\text { consultations during } & \text { COVID-19 } \\
\text { pandemic } & \end{array}$ & SCOPUS & EUA \\
\hline 17 & $\begin{array}{l}\text { Journal of the } \\
\text { American } \\
\text { Medical } \\
\text { Informatics } \\
\text { Association }\end{array}$ & $\begin{array}{l}\text { Reeves, } \\
\text { al. }^{(27)}\end{array}$ & $\begin{array}{l}\text { Rapid Response to COVID-19: } \\
\text { Health Informatics Support for } \\
\text { Outbreak Management in an } \\
\text { Academic Health System. }\end{array}$ & MEDLINE & EUA \\
\hline 18 & $\begin{array}{l}\text { Applied } \\
\text { clinical } \\
\text { informatics }\end{array}$ & $\begin{array}{l}\text { Grange, et } \\
\text { al. }{ }^{(28)}\end{array}$ & $\begin{array}{l}\text { Responding to COVID-19: The } \\
\text { UW }^{\mathbb{U l}} \text { Medicine Information } \\
\text { Technology Services Experience }\end{array}$ & WOS & EUA \\
\hline
\end{tabular}




\begin{tabular}{|c|c|c|c|c|c|}
\hline 19 & $\begin{array}{l}\text { American } \\
\text { Journal of } \\
\text { Otolaryngolog } \\
y\end{array}$ & Meng, et al. ${ }^{(29)}$ & $\begin{array}{l}\begin{array}{l}\text { Smartphone-enabled } \\
\text { otoscope-assisted }\end{array} \\
\text { telemedicine during the COVID-19 } \\
\text { outbreak }\end{array}$ & SCOPUS & China \\
\hline 20 & Oral oncology & $\begin{array}{l}\text { Machado, et } \\
\text { al. }{ }^{(30)}\end{array}$ & $\begin{array}{l}\text { Social media and telemedicine for } \\
\text { oral diagnosis and counselling in } \\
\text { the COVID-19 era }\end{array}$ & SCOPUS & Brasil \\
\hline 21 & Urology & $\begin{array}{l}\text { Gadzinski, et } \\
\text { al. }{ }^{(31)}\end{array}$ & $\begin{array}{l}\text { Telemedicine and eConsults for } \\
\text { Hospitalized Patients During } \\
\text { COVID-19. }\end{array}$ & MEDLINE & EUA \\
\hline 22 & $\begin{array}{l}\text { Clinical and } \\
\text { experimental } \\
\text { dermatology }\end{array}$ & $\begin{array}{l}\text { Marasca, et } \\
\text { al. }^{(32)}\end{array}$ & $\begin{array}{l}\text { Telemedicine and support groups } \\
\text { in order to improve the adherence } \\
\text { to treatment and health related } \\
\text { quality of life in patients affected } \\
\text { by inflammatory skin conditions } \\
\text { during COVID-19 emergency. }\end{array}$ & MEDLINE & Itália \\
\hline 23 & $\begin{array}{l}\text { European } \\
\text { Journal of } \\
\text { Physical and } \\
\text { Rehabilitation } \\
\text { Medicine }\end{array}$ & $\begin{array}{l}\text { Negrini, } \\
\text { al. }{ }^{(33)}\end{array}$ & $\begin{array}{l}\text { Telemedicine from research to } \\
\text { practice during the pandemic. } \\
\text { "Instant paper from the field" on } \\
\text { rehabilitation answers to the } \\
\text { COVID-19 emergency. }\end{array}$ & MEDLINE & Itália \\
\hline 24 & $\begin{array}{l}\text { Facial Plastic } \\
\text { Surgery \& } \\
\text { Aesthetic } \\
\text { Medicine. }\end{array}$ & $\begin{array}{l}\text { Shokri, } \\
\text { Lighthall }^{(34)}\end{array}$ & $\begin{array}{l}\text { Telemedicine in the Era of the } \\
\text { COVID-19 Pandemic: Implications } \\
\text { in Facial Plastic Surgery }\end{array}$ & WOS & EUA \\
\hline 25 & $\begin{array}{l}\text { Indian Journal } \\
\text { of } \\
\text { Community } \\
\text { Health }\end{array}$ & $\begin{array}{l}\text { Saxena, et } \\
\text { al. }{ }^{(35)}\end{array}$ & $\begin{array}{l}\text { Telemedicine units for COVID-19: } \\
\text { An experience from madhya } \\
\text { Pradesh }\end{array}$ & SCOPUS & Índia \\
\hline 26 & Urology & $\begin{array}{l}\text { Luciani, et } \\
\text { al. }{ }^{(36)}\end{array}$ & $\begin{array}{l}\text { Teleurology in the Time of } \\
\text { COVID-19 Pandemic: Here to } \\
\text { Stay? }\end{array}$ & SCOPUS & Itália \\
\hline 27 & $\begin{array}{l}\text { Telemedicine } \\
\text { and e-Health }\end{array}$ & Ren, et al. ${ }^{(37)}$ & $\begin{array}{l}\text { The Application of Mobile } \\
\text { Telehealth System to Facilitate } \\
\text { Patient Information Presentation } \\
\text { and Case Discussion }\end{array}$ & WOS & China \\
\hline 28 & $\begin{array}{l}\text { Telemedicine } \\
\text { and e-Health }\end{array}$ & Zhou, et al. ${ }^{(38)}$ & $\begin{array}{l}\text { The Distance Teaching Practice of } \\
\text { Combined Mode of Massive Open } \\
\text { Online Course Micro-Video for } \\
\text { Interns in Emergency Department } \\
\text { During the COVID-19 Epidemic } \\
\text { Period }\end{array}$ & WOS & China \\
\hline 29 & Head \& Neck & Dharmarajan, & Transition & MEDLINE & EUA \\
\hline
\end{tabular}




\begin{tabular}{|l|l|l|l|l|l|}
\hline & et al. ${ }^{(39)}$ & $\begin{array}{l}\text { multidisciplinary tumor board } \\
\text { during the COVID-19 pandemic: } \\
\text { University of Pittsburgh } \\
\text { experience. }\end{array}$ & & \\
\hline 30 & $\begin{array}{l}\text { Journal of the } \\
\text { American } \\
\text { Medical } \\
\text { Informatics } \\
\text { Association }\end{array}$ & $\begin{array}{l}\text { Perez-Alba, et } \\
\text { al. }^{(40)}\end{array}$ & $\begin{array}{l}\text { Use of self-administered surveys } \\
\text { through QR }{ }^{* *} \text { code and same center } \\
\text { telemedicine in a walk-in clinic in } \\
\text { the era of COVID-19 }\end{array}$ & México \\
\hline 31 & $\begin{array}{l}\text { Journal of } \\
\text { Nuclear } \\
\text { Medicine }\end{array}$ & $\begin{array}{l}\text { Quek, } \\
\text { Kannivelu, } \\
\text { Pua }\end{array}$ & $\begin{array}{l}\text { Yttrium-90 Radioembolization: } \\
\text { Telemedicine during COVID-19 } \\
\text { outbreak, opportunity for prime } \\
\text { time. }\end{array}$ & MEDLINE & China \\
\hline
\end{tabular}

${ }^{*}$ MEDLINE $=$ Medical Literature Analysis and Retrieval System Online; ${ }^{\dagger}$ EUA = Estados Unidos

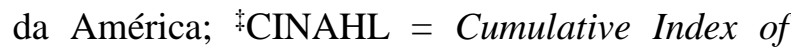
Nursing and Allied Health Literature; ${ }^{\S} \mathrm{HIV}=$ Human Immunodeficiency Virus; "WOS = Web of Science; ${ }^{\mid l} \mathrm{UW}=$ University of Washington; ${ }^{* *} \mathrm{QR}$ = Quick Response

\section{DISCUSSÃO}

\section{Recursos tecnológicos}

Os artigos incluídos na revisão apontam recursos tecnológicos que foram utilizados durante a pandemia de COVID-19, como videochamada ${ }^{(6,8,15-19,23,25-26,28,31,34)}$, ligação telefônica ${ }^{(14,22,24,36)}, \quad$ vídeo $^{(38)} \quad$ e videoconferência $^{(33,39,41)}$, prontuário eletrônico e sistema para discussão de $\operatorname{casos}^{(27,37)}$, plataforma de monitoramento ${ }^{(14,35)}$, sistema de triagem $^{(8,40)}$, rede social $^{(30)}$, formulário online para monitoramento ${ }^{(20)}$, mensagens de texto/SMS ${ }^{(32)}$, otoscópio guiado por smartphone para teleconsultas ${ }^{(29)}$, plataforma móvel para ensino ${ }^{(13)}$ e plataforma de teleconsultas ${ }^{(21)}$.

Algumas plataformas comumente utilizadas para fins sociais e de comunicação nacional e internacional foram empregadas como ferramentas de acesso às consultas de telessaúde, como o WhatsApp ${ }^{\circledR(30,32)}$, do Skype ${ }^{{ }^{(17,40)}}$ e da plataforma Zoom $\AA^{(18,26,34)}$, que apresentaram eficácia tanto na comunicação entre profissionais através de vídeos e mensagens de texto, como com os usuários que buscaram o atendimento remoto por videoconferência.

Além disso, vale destacar a utilização de estratégias próprias de algumas instituições para monitoramento e triagem de pacientes que necessitaram de acompanhamento durante o isolamento social, como foi o caso da adaptação do otoscópio para as consultas otológicas por vídeo $^{(29)}$ e a utilização de plataformas próprias para as consultas de telessaúde e a discussão dos casos pela equipe multiprofissional. Algumas ferramentas também citadas foram o FaceTime ${ }^{{ }^{(17)}}$ e o Microsoft Teams ${ }^{\circledR}{ }^{(39)}$, que possuem funcionalidades semelhantes aos anteriormente citados e possibilitam a realização de reuniões com a equipe. 


\section{Potencial e beneficios}

A telessaúde tem $\mathrm{o}$ potencial de armazenar e encaminhar dados importantes do paciente, como transferência de imagens estáticas ou vídeos armazenados, que podem ser transmitidos ao consultor receptor para análise posterior. Esse tipo de consulta pode ser mais apropriado quando implementado em cenários não urgentes ou acompanhamento de rotina do paciente $^{(32,34,40)}$, Esta questão pode estar relacionada à mudança no contexto de assistência devido à diminuição do quantitativo dos profissionais em atuação e a grande demanda de atendimento.

A insuficiência do quantitativo de profissionais da saúde suscita preocupações sobre a qualidade e a prestação de cuidados. Esse fato predispõe a expansão da utilização dos recursos da telessaúde (chats, vídeo chamadas, vídeo conferências, entre outros) no enfrentamento à COVID-19 ${ }^{(8)}$ no sentido de reintegrar aos serviços de saúde os profissionais que foram colocados em quarentena em casa após a exposição à COVID19, e possibilitar que esses profissionais em quarentena se comuniquem com os pacientes remotamente via computador ou outro recurso tecnológico, desde que esta prática não prejudique o profissional e a assistência ao paciente ${ }^{(8)}$.

Por outro lado, a participação ativa no automonitoramento de pacientes com sintomas leves não apenas melhora a iniciativa do paciente, mas também ajuda a compensar o sistema de saúde sobrecarregado ${ }^{(20,36,40)}$. Pode-se argumentar que o acesso ambulatorial durante uma pandemia deve ser ainda mais diminuído ou cancelado e isso pode acontecer, com limitação de danos potenciais à assistência, estendendo o uso da tecnologia (dispositivos móveis e computadores habilitados para webcam) nessa configuração ${ }^{(36)}$, considerando que muitos pacientes possuem smartphones ou dispositivos pessoais e eles podem ser aproveitados ${ }^{(26)}$.

A telessaúde também tem o potencial de ampliar o alcance dos cuidados a grupos em situação de vulnerabilidade durante a crise da COVID-19, como as minorias sexuais e de gênero. Em um estudo, os entrevistados estavam otimistas sobre os benefícios da telessaúde, incluindo maior acessibilidade aos prestadores de serviços de saúde e acesso mais conveniente aos serviços de saúde em geral $^{(18)}$. Os pacientes apreciam a continuidade de seus cuidados de saúde durante um período em que o acesso a serviços clínicos é reduzido, principalmente para a prevenção e assistência ao HIV. Pacientes e provedores relatam altos níveis de satisfação com a telessaúde, e os provedores podem superar a maioria dos obstáculos à prestação de serviços clínicos $^{(18)}$.

Os principais benefícios consistem em: permite que os profissionais da saúde mantenham a monitoração do atendimento ao paciente e socialização dos dados clínicos; organização antecipada para recebimento de pacientes graves e que necessite de intervenções imediatas; atendimento seguro, eficiente, eficaz e rápido ao paciente; menos exposição do profissional a agente infecciosos, como o SARS COV-2; menor lotação dos serviços de saúde; diminuição na utilização de insumos e matérias de proteção individual, devido não haver contato físico; menor custo com os valores cobrados pelos serviços de saúde, tornar o paciente protagonista do autocuidado, entre outros ${ }^{(6,8,13-15,20,26,31)}$.

Além disso, as consultas através das ferramentas disponibilizadas pela telessaúde 
promovem o acompanhamento de rotina dos pacientes que apresentam condições de saúde específicas, como cardiopatias ${ }^{(15)}$, neuropatias e pacientes oncológicos ${ }^{(27)}$ que necessitam de assistência contínua para verificação da terapêutica medicamentosa e clínica adotada, impossibilitados de comparecer as consultas durante a pandemia da COVID-19 por se enquadrarem nos grupos de risco ou por apresentarem impossibilidades em razão do estado de saúde apresentado, como também de usuários com demandas que podem ser resolvidas sem o direcionamento presencial as unidades de saúde.

\section{Desafios e obstáculos}

Um dos maiores desafios para os provedores de serviço de telessaúde é determinar quais pacientes são candidatos ideais. Ao passo que o progresso tecnológico torna possível realizar um autogerenciamento de sua saúde, um estudo apontou, no entanto, que em algumas áreas rurais remotas, o uso de smartphones e internet permanece restrito ${ }^{(29)}$. Além disso, a intervenção proposta não era conveniente o suficiente para pessoas idosas.

De fato, continuam a existir barreiras à ampla consolidação de tais serviços, incluindo o acesso restrito à internet sem conexão adequada ou sinal, aparelhos que possuam câmera e áudio de má qualidade, falta de privacidade para consulta, impossibilidade de realizar alguns métodos propedêuticos, como a palpação, ausculta e percussão, e falta de habilidade das pessoas em saber utilizar os recursos tecnológicos ${ }^{(17-18,29)}$. Reforça-se ainda que os custos de equipamentos, treinamento e licenciamento de fornecedores, além de serviços regulatórios e de pagamento são entraves presentes ${ }^{(29,34,41)}$.
Além disso, devem ser consideradas e adotadas medidas para garantir a segurança das informações do paciente, por meio de segurança de rede, segurança de dados e segurança do usuário $^{(37)}$.

Outro obstáculo importante é equilibrar a necessidade de se concentrar na pandemia e, ao mesmo tempo, manter assistência médica e operações de alta qualidade não relacionadas à nova infecção, a fim de prestar serviços a todos os aspectos da comunidade ${ }^{(27)}$.

O contexto da telessaúde é inovador e promissor, mas perpassa muitas dificuldades que precisam ser observadas e indagadas $^{(26)}$. Entretanto, deve-se atentar que a telessaúde não deverá substituir o atendimento presencial, pois a prestação de cuidados a saúde necessita do toque humano e da proximidade entre prestadores dos serviços de saúde e usuários ${ }^{(8,35)}$.

A praticidade que a telessaúde proporciona traz consigo implicações éticas e legais que devem ser ponderadas pelos prestadores dos serviços de saúde, como segurança e confidencialidade, responsabilidade profissional, arquétipos técnicos no registro, armazenamento e transmissão de dados clínicos em formato digital, direitos autorais, autorização dos órgãos fiscalizadores do exercício profissional e licença para atuação profissional remota ${ }^{(40,42)}$. Ademais, levanta-se a questão de saber se essas plataformas estão em conformidade com a ética e a regulamentação geral sobre proteção de $\operatorname{dados}^{(27,36-37)}$.

\section{Estratégias de implementação}

Para as estratégias da telessaúde serem implementadas de forma efetiva é importante salientar a necessidade de adaptações às 
realidades locais, pois cada local possui suas singularidades que podem dificultar ou potencializar suas possibilidades.

Neste contexto, os profissionais precisam utilizar as estratégias que se adequam a necessidade do paciente e que possam ser ofertadas/implementadas pelos serviços de saúde $\mathrm{e}^{(16,20,26)}$. Diante disto, os profissionais precisam e podem inovar na assistência à saúde aplicando diversos recursos de comunicação em massa, como a utilização de redes sociais para produzirem conteúdos interativos e dinâmicos como Facebook®, Twitter ${ }^{\circledR}$, Instagram $\AA ;$ utilizar plataformas de conteúdo, como Youtube®, para produzir vídeos e outros materiais; desenvolvimento de aplicativos e softwares que sejam validados para passar informações adequadas e seguras aos pacientes, entre outros recursos.

No presente estudo foi encontrada escassa produção que respondesse à pergunta norteadora no idioma português, talvez porque os profissionais e pesquisadores não estejam divulgando suas experiências, bem como estudos com baixo nível de evidência.

\section{CONCLUSÃO}

A síntese dos resultados encontrados nesse estudo revela que a telessaúde é uma estratégia efetiva utilizada por diversas áreas e especialidades da assistência à saúde para enfrentamento ao COVID-19. É válido salientar que a telessaúde é uma ferramenta transformadora para planejar, implementar e avaliar ações de enfrentamento de eventos em saúde em contextos de pandemia e de doenças infecto contagiosas.

A revisão do conhecimento indicou a necessidade de intensificar esforços para a implementação da telessaúde no enfrentamento de eventos agudos, tendo em vista que essa intervenção pode modificar a atuação dos prestadores de cuidados, utilização dos recursos públicos, materiais e insumos e proporcionando uma atuação mais eficiente e eficaz diante de pandemias e doenças infectocontagiosas. Evidenciou-se também a necessidade de estudos com métodos mais consistentes para mostrar a efetividade das intervenções implementadas, como por exemplo, ensaios clínicos randomizados e estudos de custo efetividade sobre as estratégias implementadas.

Urge a necessidade da implantação de serviços de telessaúde por outras áreas da saúde, pois no presente estudo ficou evidente que a maior produção se concentra na área da medicina. Os demais profissionais precisam aderir práticas inovadoras no que tange à assistência as pessoas, tendo em vista que as necessidades dos pacientes perpassam uma interdisciplinaridade de saberes que visa manter a prestação de cuidados de forma integral e biopsicossocial.

\section{REFERÊNCIAS}

1. Al-Shamsi HO, Alhazzani W, Alhuraiji A, Coomes EA, Chemaly RF, Almuhanna M, et al. A Practical Approach to the Management of Cancer Patients During the Novel Coronavirus Disease 2019 (COVID-19) Pandemic: An International Collaborative Group. The Oncologist [Internet]. 2020 [acesso em 2020 May 22]; 25:1-10. Available from:

https://theoncologist.onlinelibrary.wiley.com/ doi/abs/10.1634/theoncologist.2020-0213

2. World Health Organization, Pan American Health Organization. Folha informativa COVID-19 (doença causada pelo novo coronavírus) [Internet]. Brasilia: Pan American Health Organization [acesso em 2020 May 29]. Available from: https://www.paho.org/bra/index.php?option 
$=$ com_content $\&$ view $=$ article $\& i d=6101:$ covi d19\&Itemid $=875$

3. World Health Organization. Weekly epidemiological update - 2 February 2021 [Internet]. 2020 [acesso em $2021 \mathrm{Fev}$ 02]. Disponível em: https://www.who.int/publications/m/item/we ekly-epidemiological-update---2-february2021

4. Dias VMCH, Cunha CA, Vidal CFL, Corradi MFDB, Michelin L, Muglia V, et al. Guidelines on the Diagnosis, Treatment and Isolation of Patients with COVID-19. J Infect Control [Internet]. 2020 Apr [acesso em 2020 May 27];9(2). Disponível em: http://jic-

abih.com.br/index.php/jic/article/view/295

5. Sprung CL, Joynt GM, Christian MD, Truog RD, Rello J, Nates JL. Adult ICU Triage During the Coronavirus Disease 2019 Pandemic: Who Will Live and Who Will Die? Recommendations to Improve Survival. Crit Care Med [Internet]. 2020 May [acesso em 2020 Mai 13];[about 7 p.]. Disponível em: https://www.ncbi.nlm.nih.gov/pmc/articles/P MC7217126/ Epub ahead of print

6. Triantafillou V, Rajasekaran K. A Commentary on the Challenges of Telemedicine for Head and Neck Oncologic Patients during COVID-19: Otolaryngol Neck Surg [Internet]. 2020 Apr [acesso em 2020 May 13]; [about 2 p.]. Disponível em: https://journals.sagepub.com/doi/10.1177/01 94599820923622 Epub ahead of print

7. Soares FMM, Mesquita KKB, Andrade CHF de, Feitosa DSLL, Rebouças TO, Marques PGF, et al. Fatores associados à vulnerabilidade da não adesão do distanciamento social de trabalhadores na COVID-19. Revista Enfermagem Atual In Derme [Internet]. 2020 [acesso em 2021 May 14];93:e020003. Disponível em: https://revistaenfermagematual.com.br/index .php/revista/article/view/772

8. Moazzami B, Razavi-Khorasani N, Dooghaie Moghadam A, Farokhi E, Rezaei N. COVID-19 and telemedicine: Immediate action required for maintaining healthcare providers well-being. J Clin Virol [Internet]. 2020 Mai 1 [acesso em 2020 May
13];126:104345. Disponível em: http://www.sciencedirect.com/science/article /pii/S1386653220300871

9. World Health Organization. MHealth: new horizons for health through mobile technologies [Internet]. Geneva: World Health Organization; 2011 [acesso em 4 Jun 2020]. Disponível em: http://www.who.int/goe/publications/goe_m health_web.pdf

10. Peters MDJ, Godfrey C, McInerney $\mathrm{P}$, Munn Z, Tricco AC, Khalil, H. Chapter 11: Scoping Reviews (2020 version). In: Aromataris E, Munn Z (Editors). JBI Manual for Evidence Synthesis, JBI, 2020. Disponível em: https://synthesismanual.jbi.global. https://doi.org/10.46658/JBIMES-20-12

11. Tricco AC, Lillie E, Zarin W, O'Brien KK, Colquhoun H, Levac D, et al. PRISMA Extension for Scoping Reviews (PRISMAScR): Checklist and Explanation. Ann Intern Med [Internet]. 4 de setembro de 2018 [acesso em 2020 Ago 21];169(7):467-73. Disponível em: https://www.acpjournals.org/doi/10.7326/M $18-0850$

12. Akobeng A. Principles of evidence based medicine. Arch Dis Child [Internet]. 2005 Aug [acesso em 2020 Apr 29];90(8):837-40. Disponível em: https://www.ncbi.nlm.nih.gov/pmc/articles/P MC1720507/

13. Zamberg I, Manzano S, Posfay-Barbe K, Windisch O, Agoritsas T, Schiffer E. A Mobile Health Platform to Disseminate Validated Institutional Measurements During the COVID-19 Outbreak: Utilization-Focused Evaluation Study. JMIR Public Health Surveill [Internet]. 2020 Apr 14 [acesso em 2020 May 13];6(2):e18668. Disponível em: https://www.ncbi.nlm.nih.gov/pmc/articles/P MC7159056/

14. Scotté F, Minvielle E, Mir O, André F, Barlesi F, Soria J-C. A patient reported outcome platform, a useful tool to improve monitoring and effective management of Covid-19-positive patients with cancer. Eur J Cancer [Internet]. 2020 Jun [acesso em 2020 May 13];132:1-4. Disponível em: 
https://www.ncbi.nlm.nih.gov/pmc/articles/P MC7141484/

15. Knopf A. Addiction telemedicine comes into its own with COVID-19. Alcohol Drug Abuse Wkly [Internet]. 2020 [acesso em 2020 May 13];32(13):5-6. Disponível em: https://onlinelibrary.wiley.com/doi/abs/10.1 002/adaw.32673

16. Mann DM, Chen J, Chunara R, Testa PA, Nov O. COVID-19 transforms health care through telemedicine: evidence from the field. J Am Med Inform Assoc [Internet]. 2020 [acesso em 2020 May 13];1-4. Disponível em: https://academic.oup.com/jamia/advancearticle/doi/10.1093/jamia/ocaa072/5824298

17. Loeb AE, Rao SS, Ficke JR, Morris CD, Riley LHI, Levin AS. Departmental Experience and Lessons Learned With Accelerated Introduction of Telemedicine During the COVID-19 Crisis. J Am Acad Orthop Surg [Internet]. 2020 [acesso em 2020 May 13];28(11):e469-76. Disponível em:

https://journals.lww.com/jaaos/Abstract/900 0/Departmental_Experience_and_Lessons_L earned_With.99128.aspx

18. Rogers BG, Coats CS, Adams E, Murphy M, Stewart C, Arnold T, et al. Development of Telemedicine Infrastructure at an LGBTQ+ Clinic to Support HIV Prevention and Care in Response to COVID-19, Providence, RI. AIDS Behav [Internet]. 2020 Apr [acesso em 2020 May 13];1-5. Disponível em: https://www.ncbi.nlm.nih.gov/pmc/articles/P MC7189360/

19. Pollock K, Setzen M, Svider PF. Embracing telemedicine into your otolaryngology practice amid the COVID-19 crisis: An invited commentary. Am J Otolaryngol [Internet]. 2020 Apr 15 [acesso em 2020 May 13];[about 34 p.]. Disponível em: https://www.ncbi.nlm.nih.gov/pmc/articles/P MC7159874/Epub ahead of print

20. Huang S, Xiao Y, Yan L, Deng J, He M, Lu $\mathrm{J}$, et al. Implications for Online Management: Two Cases with COVID-19. Telemed E-Health [Internet]. 2020 Apr [acesso em 2020 Mai 13];26(4):487-94. Disponível https://www.liebertpub.com/doi/10.1089/tmj .2020 .0066

21. Gong K, Xu Z, Cai Z, Chen Y, Wang Z. Internet Hospitals Help Prevent and Control the Epidemic of COVID-19 in China: Multicenter User Profiling Study. J Med Internet Res [Internet]. 2020 [acesso em 2020 May 13];22(4):e18908. Disponível em: https://www.jmir.org/2020/4/e18908/

22. Borchert A, Baumgarten L, Dalela D, Jamil M, Budzyn J, Kovacevic N, et al. Managing Urology Consultations During COVID-19 Pandemic: Application of a Structured Care Pathway. Urology [Internet]. 2020 Apr 21 [acesso em 2019 May 28];[about 2 p.]. Disponível em: https://www.goldjournal.net/article/S00904295(20)30395-2/pdf Epub ahead of print.

23. Daruich A, Martin D, Bremond-Gignac D. Ocular manifestation as first sign of Coronavirus Disease 2019 (COVID-19): Interest of telemedicine during the pandemic context. J Fr Ophtalmol [Internet]. 2020 Apr 17 [acesso em 2020 May 13];43(5):389-91. Disponível em: http://www.sciencedirect.com/science/article /pii/S0181551220301480

24. Korr KS. On the Front Lines of Primary Care during the Coronavirus Pandemic Shifting from office visits to telephone triage, telemedicine. R I Med J [Internet]. 2020 Apr [acesso em 2020 May 13];103(3):9-10. Disponível em: https://search.proquest.com/openview/c4ce0 762606b1f9c2970cbd51be6de8b/1?pqorigsite $=$ gscholar $\& \mathrm{cbl}=24126$

25. Al Kasab S, Almallouhi E, Holmstedt CA. Optimizing the Use Of Teleneurology During the COVID-19 Pandemic. Telemed E-Health [Internet]. 2020 Apr 24 [acesso em 2020 May 13];[about 2 p.]. Disponível em: https://www.liebertpub.com/doi/10.1089/tmj .2020.0109 Epub ahead of print

26. Humphreys J, Schoenherr L, Elia G, Saks NT, Brown C, Barbour S, et al. Rapid Implementation of Inpatient Telepalliative Medicine Consultations During COVID-19 Pandemic. J Pain Symptom Manage [Internet]. 2020 Apr 10 [acesso em 2020 May 13];[about 6 p.]. Disponível em: 
https://www.jpsmjournal.com/article/S08853924(20)30195-0/abstract

27. Reeves JJ, Hollandsworth HM, Torriani FJ, Taplitz R, Abeles S, Tai-Seale M, et al. Rapid Response to COVID-19: Health Informatics Support for Outbreak Management in an Academic Health System. J Am Med Inform Assoc JAMIA [Internet]. 2020 Mar 24 [acesso em 2020 May 13];[about 22 p.]. Disponível em: https://europepmc.org/article/med/32208481 Epub ahead of print

28. Grange ES, Neil EJ, Stoffel M, Singh AP, Tseng E, Resco-Summers $\mathrm{K}$, et al. Responding to COVID-19: The UW Medicine Information Technology Services Experience. Appl Clin Inform [Internet]. 2020 Mar [acesso em 2020 May 13];11(2):265-75. Disponível em: http://www.thiemeconnect.de/DOI/DOI?10.1055/s-00401709715

29. Meng X, Dai Z, Hang C, Wang Y. Smartphone-enabled wireless otoscopeassisted online telemedicine during the COVID-19 outbreak. Am J Otolaryngol [Internet]. 2020 Apr 4 [acesso em 2020 May 13];[about 11 p.]. Disponível em: https://www.ncbi.nlm.nih.gov/pmc/articles/P MC7128762/ Epub ahead of print

30. Machado RA, de Souza NL, Oliveira RM, Martelli Júnior H, Bonan PRF. Social media and telemedicine for oral diagnosis and counselling in the COVID-19 era. Oral Oncol [Internet]. 2020 Apr 10 [acesso em 2020 Maio 13]; [about 2 p.]. Disponível em: https://www.ncbi.nlm.nih.gov/pmc/articles/P MC7151276/ Epub ahead of print

31. Gadzinski AJ, Andino JJ, Odisho AY, Watts KL, Gore JL, Ellimoottil C. Telemedicine and eConsults for Hospitalized Patients During COVID-19. Urology [Internet]. 2020 Apr 21 [acesso em 2020 May 13];];[about 3 p.]. Disponível em: https://www.ncbi.nlm.nih.gov/pmc/articles/P MC7172813/ Epub ahead of print

32. Marasca C, Ruggiero A, Fontanella G, Ferrillo M, Fabbrocini G, Villani A. Telemedicine and support groups in order to improve the adherence to treatment and health related quality of life in patients affected by inflammatory skin conditions during COVID-19 emergency. Clin Exp Dermatol [Internet]. 2020 [acesso em 2020 May 13];[about 1 p.]. Disponível em: https://onlinelibrary.wiley.com/doi/abs/10.1 111/ced.14245 Epub ahead of print

33. Negrini S, Kiekens C, Bernetti A, Capecci $\mathrm{M}$, Ceravolo MG, Lavezzi $\mathrm{S}$, et al. Telemedicine from research to practice during the pandemic. "Instant paper from the field" on rehabilitation answers to the Covid-19 emergency. Eur J Phys Rehabil Med [Internet]. 2020 Apr 24 [acesso em 2020 May 13];];[about 9 p.]. Disponível em: https://www.minervamedica.it/en/journals/e uropamedicophysica/article.php?cod=R33Y9999 N00A20042407 Epub ahead of print

34. Shokri T, Lighthall JG. Telemedicine in the Era of the COVID-19 Pandemic: Implications in Facial Plastic Surgery. Facial Plast Surg Aesthetic Med [Internet]. 2020 [acesso em 2020 May 13];22(5):155-6. Disponível em: https://www.liebertpub.com/doi/10.1089/fps am.2020.0163

35. Saxena S, Srivastava K, Dilbagi R, Saxena A. Telemedicine units for COVID-19: An experience from Madhya Pradesh. Indian J Community Health [Internet]. 2020 Apr [acesso em 2020 May 13];32(2):277-80. Disponível em: https://www.iapsmupuk.org/journal/index.p hp/IJCH/article/view/1452

36. Luciani LG, Mattevi D, Cai T, Giusti G, Proietti S, Malossini G. Teleurology in the Time of Covid-19 Pandemic: Here to Stay? Urology [Internet]. 2020 [acesso em 2020 May 13];140:4-6. Disponível em: https://www.ncbi.nlm.nih.gov/pmc/articles/P MC7153527/

37. Ren X, Zhai Y, Song X, Wang Z, Dou D, Li Y. The Application of Mobile Telehealth System to Facilitate Patient Information Presentation and Case Discussion. Telemed E-Health [Internet]. 2020 Apr 16 [acesso em 2020 May 13]; [about 9 p.]. Disponível em: https://www.liebertpub.com/doi/full/10.1089 $/$ tmj.2020.0084 Epub ahead of print

38. Zhou T, Huang S, Cheng J, Xiao Y. The Distance Teaching Practice of Combined 
Mode of Massive Open Online Course Micro-Video for Interns in Emergency Department During the COVID-19 Epidemic Period. Telemed E-Health [Internet]. 2020 Apr [acesso em 2020 May 13];26(5):584-8. Disponível em: https://www.liebertpub.com/doi/10.1089/tmj .2020 .0079

39. Dharmarajan $H$, Anderson JL, Kim S, Sridharan S, Duvvuri U, Ferris RL, et al. Transition to a virtual multidisciplinary tumor board during the COVID-19 pandemic: University of Pittsburgh experience. Head Neck [Internet]. 2020 [acesso em 2020 May 13];42(6):1310-6. Disponível em: https://onlinelibrary.wiley.com/doi/abs/10.1 002/hed.26195

40. Perez-Alba E, Nuzzolo-Shihadeh L, Espinosa-Mora JE, Camacho-Ortiz A. Use of self-administered surveys through QR code and same center telemedicine in a walk-in clinic in the era of COVID-19. J Am Med Inform Assoc [Internet]. 2020 [acesso em 2020 May 13]; [about 2 p.]. Disponível em:

https://academic.oup.com/jamia/advancearticle/doi/10.1093/jamia/ocaa054/5819556 Epub ahead of print

41. Quek LHH, Kannivelu A, Pua U. Yttrium90 Radioembolization: Telemedicine during COVID-19 outbreak, opportunity for prime time. J Nucl Med [Internet]. 2020 Apr 17 [acesso em 2020 May 13]; [about 3 p.]. Disponível em: http://jnm.snmjournals.org/content/early/202 0/04/23/jnumed.120.246389 Epub ahead of print

42. Rezende EJC, Melo M do CB de, Tavares EC, Santos A de F dos, Souza C de. Ethics and eHealth: Reflections for a Safe Practice. Rev Panam Salud Pública [Internet]. 2010 Jul [acesso em 2020 Mai 23];28:58-65. Disponível em: https://scielosp.org/article/rpsp/2010.v28n1/ $58-65 /$

Submissão: 2021-02-04

Aprovado: 2021-05-24 